\title{
The Lifespan Development of Writing
}

\author{
Review by Jessica Singer Early
}

So much of what we know about the practice, teaching, and study of writing takes place in silos, confined by the boundaries of time and space created by schools and institutions. 'We know too little about how writing develops before, during, and after schooling; too little about how a person's writing experiences relate to each other developmentally across the lifespan' ( $p$. 8). Charles Bazerman, Arthur Applebee, Virginia Berninger, Deborah Brandt, Steve Graham, Jill Jeffery, Paul Matsuda, Sandra Murphy, Deborah Wells Rowe, Mary Schleppegrell, and Kristen Wilcox's edited volume, The Lifespan Development of Writing, presents a lifespan view of writing development. This book emerges from a four-year collaboration between this small group of well-renowned writing scholars, who come together to share their expertise and understanding of what it means to learn to write, and to develop a new conceptual framework for studying writing development across the lifespan.

These editors come from diverse fields of study, including cognitive psychology, educational psychology, disability studies, neuroscience; emergent literacy; linguistic theory; second language learning; curriculum and assessment design; teacher professional development; urban education; and composition and rhetoric. The populations they examine in their research are equally diverse, and include preschool, elementary, adolescent, college and workplace writers. The essays in the volume illustrate how there is no fast and easy way to become a writer and that writing develops early in childhood and continues to grow, shift, and change throughout adulthood.

\footnotetext{
Affiliation

Arizona State University, Tempe, AZ

Email: Jessica.Early@asu.edu
} 
The goal of this collaboration, and of the volume as a whole, is to present a heuristic for lifespan writing development broad enough to encompass a range of age groups, contexts, and disciplines, so it may be taken up to stimulate future research and to support teachers of writing to 'see the developmental possibilities alive in their classrooms' (11). The fundamental questions addressed within the volume are wide ranging and consider the many dimensions, contexts, cultures, languages, which writers experience in a lifetime. Instead of focusing on one development stage or period, such as early childhood or adolescence, the editors ask questions like, 'How do life transitions (biological and social) relate to the experience of writing development?' or 'What travels and what falls away?' (p. 18). Rather than advocate specific instructional practices, $L D W$ asks us to recognize the 'complexity of writing development, its slow growth, its context sensitivity and variability, its interanimation with other processes of human development, and its susceptibility to fast-moving technological and communicative change' (p. 23).

Chapter One shares the challenges, exchanges, and decisions that went into the creation of this thinking group made up of writing scholars, along with key terms used in the book to discuss writing development. Chapter Two provides a synthesized framework for understanding writing across the lifespan, with eight core principles that make up this framework, which are meant to be used in future lines of inquiry into how writing develops across a lifespan. The main body of the volume, Chapters Three through Eight, shares contributions from the individual scholars or paired scholars working in collaboration, to share their views of individual writing development at specific points in the lifetime or in connection to specific writing contexts.

In Chapter Three, Deborah Rowe explores the variability and ordered progress of writing in early childhood using longitudinal and crosssectional data. Mary Schleppegrell and Frances Christie describe a linguistic trajectory of writing development for first and second language writers across years of schooling in Chapter Four. In Chapter Five, Virginia W. Berninger, Kria Geselowitz, and Peter Wallis explore how students' definitions of writing shift across early childhood, middle childhood, and early adolescence. In Chapter Six, Kristin Wilcox and Jill Jeffrey, using data from the National Study of Writing, illustrate how adolescents experience the obstacles and rewards for the development of their writing in their secondary school English, mathematics, science, and social studies classes. In Chapter Seven, Sandra Murphy and Mary Ann Smith argue against the idea of a uniform or standardized writing curriculum and make a case for writing curriculum and instruction which addresses the diversity of students' needs, abilities, interests, and strengths. In Chapter Eight, 
Deborah Brandt draws from qualitative in-depth interviews of a diverse group of adult writers to illustrate the diversity, stability, and unevenness of writing development in adulthood.

In Chapter Nine, Steve Graham presents a Persons in Context Model for writing development, which bridges social contextual and cognitive behavioral views of writing. In Chapter Ten, Charles Bazerman proposes an invitation for writing researchers to take up longitudinal studies of writing development to examine how individuals develop as writers across the lifespan. He invites researchers to collect rich and varied data to look at all dimensions of an individuals' writing growth and the diversity of writing pathways. The final chapter is a collaboration from all the editors and offers a summary of the principal themes and issues of writing development to be examined in future research, as well as implications of this complex and multi-dimensional view of writing for teachers, researchers, and policy makers. To that end, the volume's most important contribution is that it exposes the ways that learning to write is not a static skill, but a complex and fluid practice developed over a long time. These scholars present a long view of writing development, which is expansive in scope, inclusive of methodological approach and theoretical lens, and hopeful in vision.

Thus, taken as a whole, The Lifespan Development of Writing, has the potential to help educators and scholars see the ways writing development takes place beyond the silos of schools and institutions. The collection invites a lifespan perspective of writing, which means slowing down and 'stretching out time' in our teaching and in our studies and making our research more longitudinal. This perspective allows for the complexity of what it means to learn to write and invites us to think about how the students arriving into our classrooms or learning spaces each year, are experiencing only one piece of all that will make up their writing development in all of their years of schooling and in all of their lived experiences.

\section{Reference}

Bazerman, C., Applebee, A., Berninger, V., Brandt, D., Graham, S., Jeffery, J., Matsuda, P. K., Murphy, S., Rowe, D. W., Schleppegrell, M., and Wilcox, K. C. (2018). The Lifespan Development of Writing. Urbana, IL: NCTE Press. 
